\title{
Psicología de la violación
}

\author{
VICENTE GARRIDO \\ Universidad de Valencia

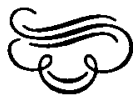

Resumen

Este artículo constituye una revisión de la literatura psicológica referida a la violación. En la primera parte nos ocupamos del agresor sexual, enfatizando la naturaleza fundamentalmente agresiva de la violación, y poniendo de manifiesto la necesidad de superar la perspectiva tipologica. La segunda parte trata de la victima, poniéndose de relieve los impactos de la agresión recibida y las difíciles circunstancias por las que pasa la mujer que ba denunciado el becho en el proceso penal. Se concluye acentuando el aspecto social de la violación, y comentando las medidas que habría que adoptar a nivel psico-social con objeto de prevenir la aparición de esta conducta.

\section{Abstract}

This paper reviews the psychological literature on rape. The first part focuses on the rapist, stressing the aggressive nature of rape and the necessity to overcome the typological approach. The second part studies the victim, underlying the impact of the aggression suffered and the difficult circumstances that follow the victim's report to police. It concludes by stressing the social side of rape and some preventive measures are suggested.

Dirección del autor: Universidad de Valencia. Facultad de Filosofia y CC. de la Educación. Departamento de Teoría de la Educación. Ava. Blasco Ibáñez, 46010 Valencia. 
En el presente artículo exponemos una revisión de la literatura psicológica más relevante relacionada con el delito de violación. Lo hemos dividido en dos partes. La primera hace referencia al agresor y la segunda a la víctima. Si bien son bastante numerosos los trabajos psicológicos referidos a tipos delictivos, éstos se han revelado como poco satisfactorios a la hora de cualificar los determinantes que inciden en el hecho. Bartol (1980) y Brodsky (1980) coinciden en afirmar que la literatura sobre tipos aporta poco a la comprensión del fenómeno delictivo. Como se verá, el peso específico que los roles y actitudes juegan en la perpetración de este acto hace sumamente difícil validar categorías discretas de delincuentes sexuales.

En el caso de la víctima, hasta hace poco más de una década desconocíamos casi todo de lo que acontecía a la mujer que había sido objeto de una agresión sexual de esta naturaleza. Sin embargo, creemos que una perspectiva «victimológica» por parte de la psicología de la delincuencia es absolutamente necesaria; no en balde el delito implica la presencia al menos de dos protagonistas. El caso de las víctimas de violación es especialmente revelador, al ponerse de relieve tanto patrones de socialización como de actuación legal en el marco del proceso que sigue a la denuncia. La profundización metodológica de este tipo de estudios puede arrojar mucha luz sobre posibles planes de prevención de la violación y asesoramiento a las víctimas.

\section{EL AGRESOR}

Brodsky (1980) ha comentado certeramente que las primeras teorías de la delincuencia creyeron que cada delito violento implicaba un tipo de personalidad o bien una anormalidad conductual. En la actualidad los psicólogos criminales no parecen ya apoyar esta idea. Path (1976), por ejemplo, ha indicado la dificultad existente para encontrar un cuadro único del violador, haciendo hincapié en la necesidad de desvelar mitos y estereotipos ampliamente difundidos en la literatura.

\section{Los mitos}

Quizá uno de los más frecuentados es el que califica al agresor sexual como neurótico. Karpman (1954), un psicoanalista que hizo célebre su obra The sexual offender and his offenses creía que los violadores padecían lo que denominó "neurosis parafilíaca», donde el elemento sexual llega a ser el condicionante fundamental de su conducta. De ahí que para Karpman el violador sea equiparable al enfermo mental $y$, por consiguiente, se halle exento de responsabilidad ante la ley.

Dejando al margen el matiz heterodoxo con respecto al pensamiento freudiano que suponía la tesis de Karpman, otros investigadores más modernamente han venido a sumarse a la consideración del agresor sexual como perturbado emocional, aunque sin la rotundidad de Karpman. Así, Groth (1977) entiende que «... el acto de la violación puede representar un síntoma de un defecto del desarrollo: un fracaso para alcanzar un adecuado sentido de la propia identidad, la consecuencia de llegar a ser especialmente grave en la adolescencia (...) la violación, en este contexto, representa una crisis interna o del desarrollo del delincuente» (págs. 253-254). 
En la actualidad no parece que la evidencia empírica apoye la idea del agresor sexual como neurótico o perturbado emocionalmente. Referido a la psicosis, Henn et al. (1976) indicaron que su incidencia en los violadores era inferior a la de la población general. Por otra parte, la etiqueta usualmente asignada a los violadores de "personalidades antisociales" no indica nada más que la existencia de una historia conductual antisocial, y no un problema emocional (Henn et al., 1976; McCaldon, 1967; Koop, 1962).

Bauermeister (1977) recoge desde distintas respectivas experiencias que hablan de la normalidad cultural (en ciertas situaciones) de la violación. Quizá la más sólida descanse en el hoy clásico libro de Brownmiller, donde se muestra el empleo de la violación como medio de agresión y humillación por parte de los vencedores en las guerras. "La actividad (violación), que ha sido practicada por la humanidad desde tiempos inmemoriales, debe estar alimentada por fuerzas psicológicas, las cuales son parte de la constitución normal biológica y social» (Bauermeister, 1977, pág. 245).

Esto nos lleva al segundo de los estereotipos mantenidos con respecto a la personalidad del agresor: el ser un «superhombre», impelido por fuertes deseos sexuales. En la actualidad tenemos suficientes datos como para asegurar que el componente fundamental de la violación no es el sexo, sino la hostilidad/poder (Osborne, 1982; Groth et al., 1977; Groth, 1979; Zoomer, 1983; Weis y Weis, 1975, entre otros muchos).

Una rápida revisión sobre las dos tipologías más extendidas servirá para ejemplificar este punto. En la tipología del Bridgewater treatment center de Massachusetts elaborada por Cohen y su equipo (Cohen et al., 1969, 1971) se distingue: 1) El violador de agresión desplazada, sin ninguna excitación sexual inicial, ya que la violación tiene el sentido de agraviar y humillar a la víctima empleando con frecuencia el sadismo. El término de esta categoría procede del hecho de que la mujer no ha jugado un rol directo en el desencadenamiento de la agresión. 2) Violador compensatorio, motivado fundamentalmente por demostrar a la víctima su competencia sexual, en un intento de compensar su falta de adecuación para una vida socialmente ajustada. 3) Violador sexual-agresivo, donde para sentir excitación sexual el atacante debe infringir daño físico. 4) Violador impulsivo, cuya acción es el resultado de aprovechar una «buena oportunidad», usualmente presente en el transcurso de otros hechos delictivos como el robo.

En la tipología de Groth (Groth et al., 1977; Groth, 1979) aparecen tres componentes de modo necesario en la psicología de los agresores sexuales: hostilidad, poder y sexualidad. Las interrelaciones entre estos factores y la intensidad relativa con que son expresados varía de un sujeto a otro. Sin embargo, la agrupación de esas dimensiones le llevó a concluir tres patrones básicos de agresión:

1) violación de hostilidad: hay más violencia de la necesaria para consumar el acto, y la erección procede de la propia exhibición de fuerza.

2) Violación de poder: la meta es la conquista sexual, como compensación a su existencia rutinaria. De nuevo parece que la satisfacción sexual alcanzada por estos sujetos es escasa, toda vez que manifiestan gran cantidad de fantasías masturbatorias.

3) En la violación sádica se funde el aspecto de sexualidad con el de poder. A diferencia del primer tipo, que supone una explosión de hostili- 
dad, el asalto aquí es totalmente premeditado, donde la agresión proporciona satisfacción sexual en un feedback a modo de espiral.

Evidentemente, estas tipologías empíricas no están exentas de dificultades. Bartol (1980) ha reclamado mayor atención para el estudio de los factores críticos que, en la historia conductual de un sujeto, condicionan una mayor probabilidad de implicarse en agresiones sexuales. Igualmente, Brodsky (1980) puntualiza que lo que necesitamos ahora es una «tipología de tipologías», es decir, "una síntesis teórica y empírica de los tipos existentes» (pág. 103).

Sea como fuere, los estudios de casos que han llevado al desarrollo de estas tipologías (véanse otras también en Bromberg y Coyle, 1974; Roebuck, 1967; Nedoma et al., 1971) han negado de modo consistente la aureola hipersexuada de la violación. Osborne (1982, pág. 80 ) lo ha expuesto claramente:

«La sexualidad, por consiguiente, puede contemplarse como algo secundario en la violación. En combinación con distintos grados de hostilidad y poder, la expresión hostil de la sexualidad es la forma en que el agresor intenta enfrentarse a las vicisitudes de la vida diaria. El detonante puede ser o no un acontecimiento estresante: las experiencias continuas de fracaso, incompetencia o rechazo pueden culminar en la manifestación de agresión o dominancia a través de la humillación sexual y el control de una víctima.

La violencia de la agresión sexual, pues, parece descansar en un patrón de conducta violenta general, no constreñible a personalidades neuróticas ni a sujetos hipersexuados. Como tal conducta violenta $-\mathrm{y}$ a falta de estadísticas específicas sobre el caso-, puede ocurrir que represente el modo de vida de las subculturas delictivas (Wolfgang, 1967), como parece desprenderse del muy citado trabajo de Amir, Patterns in Forcible Rape (1971), o de los trabajos de Kinsey, quien en 1965 puntualizó que la gran mayoría de los agresores sexuales tenían antecedentes por delitos no sexuales (Bauermeister, 1977).

Los violadores, sin embargo, parece que ostentan también un amplio historial de agresión sexual. Trabajos de Longo y Groth (Longo, 1982; Longo y Groth, 1983; Groth, 1977) han puesto de manifiesto una tendencia en esta línea, indicando además como factor de interés una posible acción traumática debida a su mayor precocidad sexual, o a los abusos sexuales sufridos en la infancia.

\section{Explicaciones}

La revisión de la bibliografía sobre la violación permite entresacar tres niveles de explicación. El primero de ellos podríamos calificarlo de intrapsíquico, psicodinámico o de la perturbación, y coincide con el primero de los estereotipos o mitos que hemos comentado anteriormente. En este modelo incluiríamos autores como Groth (1979), Barlow (1974), Abel et al., (1976), MacDonald (1974) y Rosenberg (1981). Por su parte, Annis (1982), de planteamiento más bien ecléctico, no duda en afirmar que: «Con independencia de las diferencias individuales, todo el tratamiento asume que la violación, los abusos deshonestos con niños, el incesto, el voyeurismo y el 
exhibicionismo son resultados de problemas intrapsíquicos e interpersonales, con frecuencia duraderos" (pág. 224).

No cabe duda que en este modelo incluiríamos también el ya comentado trabajo de Karpman (1954), y en general todos aquellos que busquen explicaciones a la agresión sexual por el lado del desequilibrio y/o psicopatología.

El segundo modelo o nivel explicativo sería el propiciado por la psicología de los rasgos, en donde, en contraste con la aproximación tipológica, no se buscan las diferencias entre los delincuentes, sino las similitudes, las comunalidades que pertenecen a muchos delincuentes sexuales, y que les distinguen de otros delincuentes o grupos de control (Brodsky, 1980).

Esta línea de trabajo ha encontrado de manera sistemática dos áreas. En la primera, se alude a que el agresor sexual es una persona con deficientes habilidades interpersonales, incapaz de mantener relaciones sociales y afectivas sólidas, algo que inevitablemente nos recuerda una de las principales características del psicópata (McCord, 1982). En la segunda, y quizá como consecuencia lógica, se tiende a describir al agresor sexual como carente de asertividad, de autoestima. Fisher y Rivlin (1971) investigaron cien violadores en un penal de California empleando la Escala de Edwards (E.P.P.S.). Comparados con los varones normales, los violadores tendían a aparecer como menos dominantes y menos independientes, en todo caso deseosos de mantener relaciones heterosexuales.

Estas dos áreas pueden reasumirse en lo que podemos denominar hipótesis de la inmadurez, donde se hace hincapié en la gratificación sexual fuera de los cauces apropiados. Así, Goldstein (1977) encontró que los violadores derivaban su placer de fantasías elicitadas por estímulos de los medios de comunicación social, mientras que los sujetos control preferían los encuentros reales. También los primeros exhibían una tasa mayor de masturbación que los segundos en la edad adulta, con motivo de estímulos obtenidos en dichos medios.

El tercer modelo explicativo descansa en el concepto de subcultura de la violencia, ya introducido anteriormente, tal como lo definió Wolfgang (1967). En este caso, el delito de violación no sería sino uno de los muchos que se cometen en estos settings criminógenos, donde los sujetos, firmemente anclados en una carrera delictiva, rivalizan con las conductas y valores socialmente establecidos.

La idea de que la mayoría de las violaciones son el producto de una conducta predatoria generalizada en una minoría de la población (subcultura delictiva) tiene algunos apoyos. Así, los datos que evidencian que la agresión sexual se halla acompañada de antecedentes no únicamente violentos, es una consideración a tener en cuenta. Bauermeister (1977) hace referencia a este grupo de violadores cuando se quiere referir a aquellos «normales», es decir, para los que la violación tiene el sentido de «adquirir prestigio y poder dentro del grupo de iguales. Alli donde las subculturas de la violencia apoyen al violador, como en el caso de contiendas nacionales, raciales o de clases, el violador será "normal", es decir, su conducta mostrará las características típicas de la conducta normal" (pág. 247).

Para Bauermeister los violadores anormales actuarían fuera del contexto y del apoyo de su grupo de referencia: «Donde la violación se desvía de su ambiente típico, donde no ocurre en un ambiente real de violencia, no- 
sotros podemos sospechar la existencia de una desviación individual o una psicopatologías (pág. 247).

Otros apoyos a este modelo proceden de la mayor tasa de delincuentes sexuales procedentes de la clase baja (Chappell, 1977a, citado en Bartol, 1980), de donde se extrae de manera abrumadora el mayor porcentaje de delincuentes comunes (véase una revisión de este tópico en Garrido, 1984), así como de la vinculación con el alcohol (Rada, 1975), tradicionalmente asociado por su carga elicitadora de agresividad a las subculturas de la violencia.

Finalmente, el cuarto modelo responde a un planteamiento sociológico, conformando la bipótesis de la socialización. Desde esta perspectiva, la sociedad se estructura ideológicamente de forma tal que la mujer llega a ser una "víctima legitimada" (Weis y Weis, 1975) de unos roles y estereotipos que mediatizan las relaciones y expectativas interpersonales. Así, mientras al varón se le enseña a «tomar la iniciativa» con las mujeres, a ser dominante, a enorgullecerse de las conquistas sexuales, la mujer es socializada para la búsqueda de protección del varón, para la pasividad. No es de extrañar, como luego se verá, que su rol de víctima se torne en el de delincuente con respecto al proceso penal.

\section{Evaluación de los modelos}

El modelo psicoanalítico, a decir de Bauermeister (1977), ha sido junto con la «sabiduría popular», tan apegada a los estereotipos, y la demagogia política, uno de los grandes obstáculos para comprender el fenómeno de la psicología de la violación en toda su extensión.

Podemos estar de acuerdo con el modelo de la perturbación por lo que respecta a algunos casos en donde quizá sea aplicable el término de «psicópata sexual», aunque no coincidamos en los planteamientos psicodinámicos a la hora de definir tales perturbaciones según su ortodoxia.

Rechazada la idea global de anormalidad para el agresor sexual, los otros modelos tienen a nuestro modo de ver mayor fuerza explicativa, aunque ninguno de ellos llegue por sí solo a ser suficiente.

Así, la postulación de unas deficiencias interpersonales parece coherente con los datos clínicos que poseemos, y en la actualidad muchos programas dirigidos a agresores sexuales contemplan el entrenamiento en habilidades sociales como un foco de máxima atención (Annis, 1982).

Igual razonamiento podemos esgrimir por lo que respecta al modelo de la subcultura de la violencia, aunque hayamos de hacer una precisión importante: el hecho de que la agresión sexual sea una de las posibilidades delictivas de los sujetos pertenecientes a las subculturas de la violencia, no significa que sólo ellos lleven a cabo violaciones.

Más bien hoy poseemos datos que ponen en serios aprietos esa posible especificidad de la violación. El estudio de Amir (1971) en la ciudad de Filadelfia y del Bureau de Investigación Criminal de Australia (1974), entre otros, han coincidido en señalar que en un porcentaje muy elevado de los casos $(47 \%$ y $33 \%$, respectivamente) la víctima y el agresor se conocían, eran vecinos o parientes, y nada menos que el $57 \%$ (en el estudio de Amir) y el $50 \%$ (en el de Australia) de los casos ocurrieron en casa de la víctima o del asaltante. 
Por otra parte, Scutt (1982) ha señalado la gran cantidad de violaciones ocurridas entre cónyuges, algo que en ciertos paises está penado, pero que usualmente suele desestimarse como objeto de persecución. La consideración de las violaciones en el seno de la familia entre padres e hijos elevaria sin duda más la tasa de incidencia de este delito fuera del contexto de las poblaciones tradicionalmente consideradas delictivas.

De este modo, la perspectiva socializadora o de la "víctima legitimada" ha de tomarse ineludiblemente para la comprensión cabal del fenómeno de . la violación. Desde la década pasada, son varios los autores y líneas de trabajo que inciden en la definición y fomento social de la agresión contra las mujeres. En este contexto, las teorías tradicionales de la agresión de corte individual son marcadamente insuficientes (Zoomer, 1983). En la actualidad las investigaciones más relevantes en el estudio de los agresores sexuales toman en consideración el aspecto actitudinal y sociológico, dentro de una línea de trabajo experimental y rigurosa.

\section{Sociologia y laboratorio}

Recientemente, han aparecido dos líneas de desarrollo empírico que prometen obtener resultados sugestivos en el diagnóstico y predicción de la agresión sexual. El primero de ellos se relaciona con las actitudes facilitadoras de la agresión hacia las mujeres en el terreno sexual, siendo su máximo exponente Martha R. Burt (Burt, 1980).

Burt teorizó que ciertas actitudes intrínsecas a la ideología y cultura occidental forman parte de la estructura cognoscitiva de los violadores. Su trabajo se centra fundamentalmente en lo que ella denomina las creencias o mitos de la violación, definidos como visiones estereotipadas relacionadas con la violación, las víctimas de violación y los violadores. Quizá el más representativo de tales mitos sea el que sostiene que las mujeres en realidad "piden ser violadas", de tal forma que sus quejas y acusaciones no son sino coartadas de sus deseos sexuales ocultos. Burt hipotetizó que tales creencias pueden ser empleadas como «neutralizadores o alivios psicológicos, permitiendo a los violadores potenciales eliminar las prohibiciones sociales» (1978, pág. 282, citado en Malamuth, 1983).

Con objeto de poner a prueba su teoría, Burt desarrolló varias escalas destinadas a medir varios tipos de actitudes que facilitan la agresión contra las mujeres. En un estudio de las actitudes de una muestra de Minnesota, ella halló que las respuestas que evaluaban la aceptación de los mitos de la violación se relacionaban notablemente con las respuestas dadas a una escala que media aceptación de la agresión contra la mujer.

La segunda área de trabajo tiene que ver con la responsividad sexual hacia la violación, más en concreto, con respecto a las historias que describen un suceso de violación. Abel et al. (1977) encontraron que sujetos convictos del delito de violación mostraban el mismo grado de tumescencia del pene (indicador de la respuesta sexual) ante historias de violación que ante historias de relaciones sexuales consentidas. En contraste, los sujetos control mostraban mayor activación sexual ante las historias de sexo consentido que ante las de violación. Además, Abel y colaboradores encontraron que dentro de la muestra de violadores, los niveles más altos de agresión durante la comisión de la violación se relacionaban con una más alta res- 
puesta sexual en relación a las historias de violación, pero no con las de sexo consentido.

Aunque contamos con bastante evidencia empírica apoyando los resultados de Abel y colaboradores (Abel et al., 1978; Barbaree et al., 1979; Quinsey et al., 1981), también se ha obtenido que el arousal sexual de los no violadores ante historias de violación resultaba inhibido (en relación a las historias de sexo consentido) sólo bajo ciertas circunstancias, tales como que las historias de violación mostraran el horror y sufrimiento de la víctima (Malamuth et al., 1980; Malamuth y Check, 1980a, 1980b, 1983). Por otra parte, parece que en la población general existe una minoría que se comporta igualmente ante los dos tipos de historias, es decir, de la misma forma que los violadores (Malamuth y Check, 1983).

Sobre la base de sus hallazgos, Abel et al. (1977) desarrollaron el «índice de la violación", el cual supone la medición de la tumescencia del pene relativa a las historias de violación en comparación con las historias de sexo consentido. Sin embargo, en la medida en que, como ya hemos mencionado, se ha encontrado en la población general ciertos sujetos mostrando un "indice de violación» semejante al de los violadores, ello puede indicar que estos hombres precisen de ciertas condiciones para actuar de forma sexualmente agresiva o, alternativamente, que el arousal hacia la violación es una respuesta aislada que no puede homologarse con la conducta efectiva (Malamuth, 1983).

Por otra parte, poseemos igualmente evidencia empírica de que las actitudes que facilitan la agresión contra las mujeres y arousal sexual a la violación pueden obtenerse empleando como medida la probabilidad de violar tal y como es informada por el mismo sujeto (Check y Malamuth, 1983; Malamuth, 1981a, 1981b).

Malamuth (1983) ha desarrollado recientemente una investigación para comprobar si los factores descritos arriba (actitudes facilitadoras de la violación y arousal sexual ante historias de violación) podían predecir la agresión de los hombres contra una mujer dentro de una situación de laboratorio. En fundamento teórico de su estudio se halla en la teoría que sugiere que varios actos de agresión contra las mujeres pueden compartir algunas causas similares subyacentes (Burt, 1980).

En su investigación, el autor dispuso dos fases. En la fase I se evaluaba el arousal sexual a través de la tumescencia del pene (rape index) en dos sesiones separadas por 8-9 semanas. También se evaluaron las actitudes facilitando la violencia contra las mujeres a través de dos escalas diseñadas por Brut: Rape Myth Acceptance y Acceptance of Interpersonal Violence, cinco de cuyos seis items se refieren a la violencia contra las mujeres. La fase II implicaba una situación de laboratorio, en donde el sujeto, desconocedor de la relación existente entre ambas fases, debía de administrabar estímulos aversivos a una confederada del experimentador cuando ésta cometiera una serie de errores. Previamente se habian inducido sentimientos de hostilidad del hombre para con la mujer. 69 y 42 sujetos participaron en ambas fases de la investigación, respectivamente.

Empleando un análisis de ecuaciones estructurales, donde se distinguen entre factores latentes (constructos teóricos) y variables observadas, se elaboró un modelo estructural tal y como aparece en la Figura 1. En la Tabla I se hace constar los parámetros, pesos estandarizados y razones críticas del modelo. 


\section{FIGURA 1}

Modelo estructural empleado por Malamuth (1983)

Los factores latentes se representan por circulos, y los factores observados por cuadrados. Este modelo tiene dos factores predictivos (Actitudes facilitadoras de la violencia y Excitación sexual ante la violación), losa cuales son libres de relacionarse entre si al igual que con la variable dependiente (agresión contra la mujer).

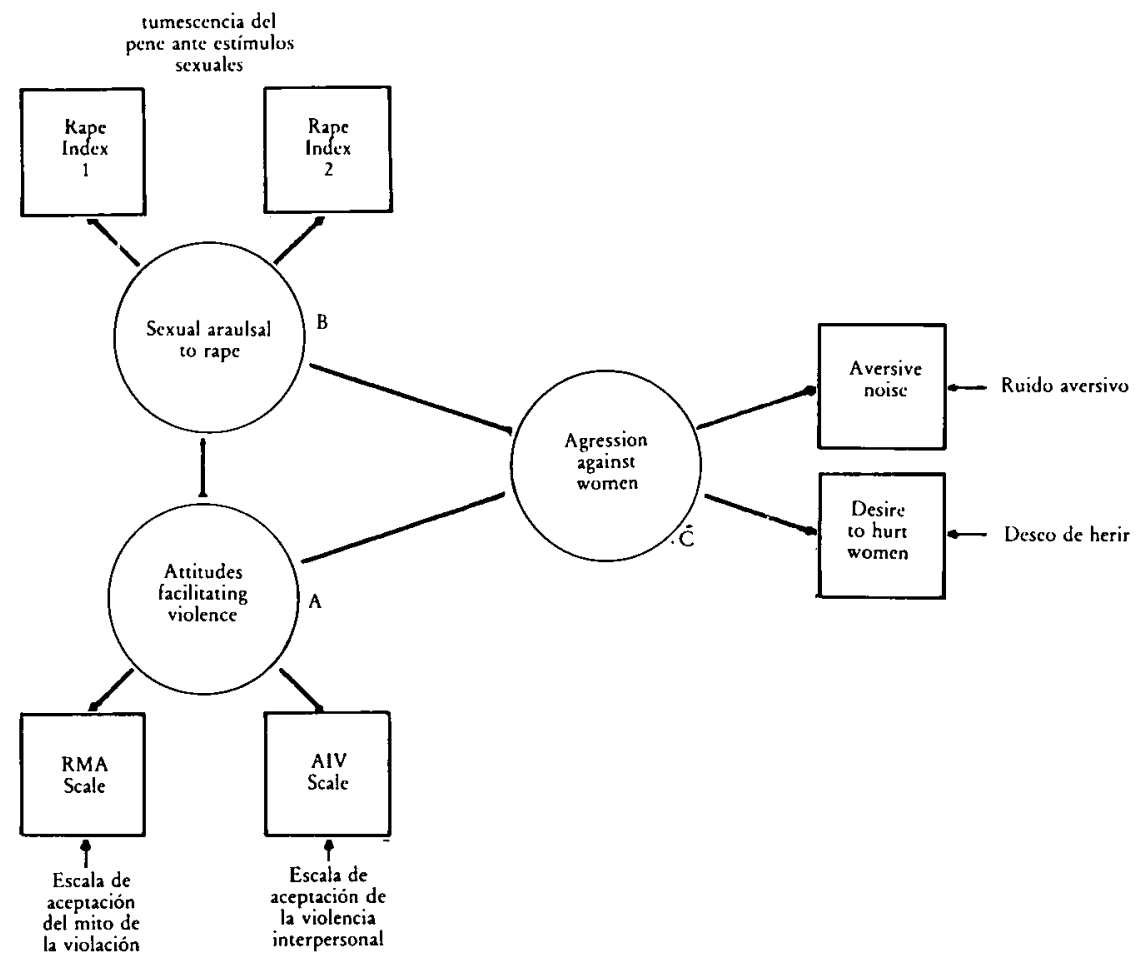

Nota: Los factores latentes se representan por círculos, y los factores observados por cuadrados. Este modelo tiene dos facNota: Los factores latentes se representan por círculos, y los factores observados por cuadrados. Este modelo tiene dos fac-
tores predictivos (Actitudes facilitadoras de la violencia y Excitación sexual ante la violación), los cuales son libres de relacionarse entre si al igual que con la variable dependiente (agresión contra la mujer).

\section{TABLA I}

Estimaciones de los parámetros estandarizados

(Malamuth, 1983)

\begin{tabular}{lcc}
\hline Factores y variables & $\begin{array}{c}\text { Peso } \\
\text { factorial }\end{array}$ & $\begin{array}{l}\text { Varianza } \\
\text { de error }\end{array}$ \\
\hline
\end{tabular}

Parámetros del modelo de medida

Actitudes facilitando la violencia (A)

Rape mith Acceptance Scale

Acceptance of Interp. Violence Sc.

Arousal Sexual hacia la violación (B)

Indice de violación 1

Agresión contra la mujer (C) 
Parámetros del modelo estructural

Correlaciones entre factores $\mathrm{A} \times \mathrm{B}$

Pesos de Regresión

$\mathrm{A} \times \mathrm{C}$

Varianza residual estandarizada

Agresión contra la mujer

0,57

Como puede verse, el análisis de regresión arousal sexual a la violación correlacionó significativamente con agresión hacia la mujer (.42), acercándose actitudes facilitando la violencia (.38) y menos, la relación entre arousal y actitudes (.32). Las dos variables independientes explicaron el $.43 \%$ de la varianza de la variable dependiente.

Los trabajos de Malamuth y de otros autores que hemos citado son relevantes desde un doble plano. Conceptualmente, porque suponen un acercamiento al fenómeno de la violación amplio, en donde el aspecto de actitudes y roles es considerado junto con variables «más duras» de tradición experimental. Metodológicamente, porque rompe con tipologías descriptivas y estudios anecdóticos de casos para acercarse a una aproximación de laboratorio, donde se ponen en juego variables previamente evaluadas como decisivas. Desde luego, no podemos extraer inferencias causales, pero el hecho de que tanto la medida fisiológica como la actitudinal contribuyeran independientemente a predecir la agresión contra la mujer, puede darnos una idea de los tipos de trabajos que posteriormente han de diseñarse.

\section{LA VICTIMA}

French y Wailes (1982) presentaron un cuestionario de elección forzada a 215 varones y 260 mujeres, blancos y de color, extraídos de 2 colleges norteamericanos. La prueba contenía la descripción de 13 actos sexuales desviados. En la ordenación de éstos, el delito de violación alcanzó el segundo puesto en los referente a la gravedad y repudio por parte de los encuestados.

Este trabajo puede ser un indicador de un cambio de actitud en las nuevas generaciones con respecto a la consideración de la violación, acorde con el mayor esfuerzo que en los últimos años viene prodigando la comunidad psicológica en la investigación de la víctima como objeto focal de estudio.

Esto es importante, ya que los estudios de la delincuencia han atendido tradicionalmente al papel del agresor, ya sea para buscar patologías de personalidad o determinantes socioambientales. Incluso la denominada sociología de la desviación, de marcado signo progresista, no se ha ocupado de las víctimas; antes bien, ha considerado a los delincuentes como víctimas de la sociedad (véase, por ejemplo, Bernard, 1981).

$\mathrm{Ni}$ siquiera la propia Victimología, desviándose del corpus de trabajo con que la definió Mendelsohn en 1947, ha sabido acercarse a la víctima 
como protagonista "perdedor» en la confrontación con el delincuente. hasta hace bien poco, en efecto, los trabajos victimológicos tendían a considerar el papel causal de la víctima en el delito (Weis y Weis, 1975).

No extrañará, pues, que hasta hace muy poco desconociéramos casi de forma absoluta lo que acontecía a las mujeres que habían sido víctimas del delito de violación. Ahora bien, con la celebración en Jerusalén del Primer Simposium Internacional de Victimología (Drapkin y Viano, 1974), se estableció el eslabón académico para el reconocimiento social de la víctima. En los múltiples trabajos presentados en dicho encuentro científico, aquéllos referidos a las víctimas de violación sirvieron de piedra angular para desarrollar investigaciones posteriores, las cuales tomaron dos líneas de acción bien definidas: 1) analizar los impactos del trauma de la violación a través de estudios de seguimiento de las mujeres afectadas, y 2) analizar el proceso penal y sus repercusiones psico-sociológicas sobre aquellas víctimas que denuncian el hecho.

Los trabajos de Burgess y Holmstrom (Burges y Holmstrom, 1974a y b; Holmstrom y Burges, 1974) supusieron sendos esfuerzos en ambas líneas, al igual que el programa del Centro de Violación del Hospital de Filadelfia de Peters (Peters, 1974), a los cuales se han sumado otros autores, bien empleando distintas pruebas stándar o específicamente diseñadas para evaluar el funcionamiento de la víctima en áreas diferentes de su conducta (Feldman et al., 1979, Norries y Feldman, 1981; Orlando y Koos, 1983), o desde una perspectiva más sociológica, considerando las variables ideológico-culturales que viene a definir e incluso a propiciar el asalto (Burt, 1980; Weis y Weis, 1975; Zoomer, 1983).

Lo que sigue es un intento por exponer de manera suscinta las principales conclusiones y preocupaciones derivadas de estas líneas de investigación.

\section{Impactos de la violación}

En este punto conviene distinguir entre la reacción de la víctima durante el asalto y después de éste. En el asalto, una respuesta común es el miedo (Burgess y Holmstrom, 1974a), e incluso el miedo a morir (Peters, 1974).

Las reacciones después del asalto han sido estudiadas como un primer paso necesario para poder idear estrategias de apoyo y asesoramiento durante las crisis derivadas de la violación. El primer trabajo de estas características se debe a Sutherland y Scherl (1970), quienes estudiaron a $13 \mathrm{mu}$ jeres de clase media, jóvenes y de raza blanca. Ellos describieron un patrón de respuesta compuesto de 3 fases.

En el primero se da una reacción de schock, con gran ansiedad y temor. La mujer se hallaba receptiva ante los demás, y la denuncia ante las autoridades en ocasiones se demoraba debido a la preocupación de cómo reaccionarían ante el hecho de que han sido víctimas. Horas o días después aparece una segunda fase, que durará semanas o meses. Aquí la ansiedad generalizada disminuye, produciéndose una adaptación al mundo exterior. La fase tercera, denominada de «integración y resolución", se caracteriza por sentimientos depresivos, pensamientos obsesivos en relación a la violación, autoinculpación y hostilidad hacia el agresor. Los autores encontraron que la víctima aquí vuelve otra vez a ser comunicativa, dándose mayor proba- 
bilidad -en la mejor ortodoxia freudiana- de examinar y resolver los problemas emocionales relacionados con el ataque (Osborne, 1982).

Pero el trabajo más destacado sobre impactos de la violación se debe a Ann Wolbert Burgess y Linda-Lyte Holmstrom. Burgess y Holmstrom (1974) entrevistaron y siguieron la evolución de 92 mujeres adultas, víctimas de la violación por la fuerza, que fueron atendidas en la sala de urgencias del Boston City Hospital.

Las autoras se personaban al lado de la víctima nada más era ingresada en el hospital, y ya permanecían en contacto con ella durante un período de un año a través de entrevistas y llamadas telefónicas. Ello les permitió esbozar un patrón típico de respuestas al que denominaron el "síndrome de trauma de violación»:

«El síndrome del trauma de violación consiste en una fase aguda y un proceso de reorganización a largo plazo, que aparece como consecuencia de una violación por la fuerza o un intento de violación. Este síndrome, con trastornos de comportamiento, somáticos y psicológicos, constituye una reacción aguda de stress ante una amenaza a la propia vida» (1979, pág. 138. Original 1974).

El síndrome consta de una primera fase de desorganización, donde la víctima en las primeras horas experimenta una amplia gama de emociones, junto a schock e incredulidad. Tales emociones podían revestir dos patrones: el de tipo expresivo, con sentimientos de pánico, ira y ansiedad, y el de tipo controlado, en el que los sentimientos se ocultaban bajo un aspecto sosegado o deprimido.

Más allá de estas reacciones de impacto aparecen unas reacciones en las primeras semanas posteriores a la violación; éstas eran: 1) trauma físico derivado del ataque, 2) tensión de la musculatura esquelética, con dolores de cabeza, fatiga y trastornos del sueño, 3) irritabilidad gastrointestinal, con náuseas, pérdida del apetito y dolores de estómago, y 4) trastornos genitourinarios.

Finalmente, en esta primera fase surgen también reacciones emocionales. La ira, el deseo de venganza y la autoculpabilización eran algunas de las más destacadas, pero el sentimiento principal era el temor a la violencia y la muerte. Las víctimas afirmaban que lo que les inquietaba no era tanto la violación, sino el sentimiento de que después de la agresión les iban a matar.

En la segunda fase o proceso a largo plazo, descrita como de reorganización, la gran mayoría de las mujeres manifestaron padecer algún tipo de alteración o trastorno en su forma de vida, aunque no todas experimentaron los mismos síntomas ni el orden de aparición de éstos fue el mismo.

En esta fase aparecían, fundamentalmente:

1) Aumento de la actividad motora, evidente sobre todo en el cambio de residencia destinado a conseguir una mayor seguridad y reemprender una nueva vida. Otra respuesta fue acudir a visitar familiares que normalmente no se frecuentaban.

2) Pesadillas, Veintinueve mujeres tuvieron sueños con elementos de terror y angustia.

3) Traumatofobia: desarrollo de fobias como reacción de defensa ante las circunstancias de la violación. Las fobias que aparecieron con mayor fre- 
cuencia fueron: miedo a estar dentro de casa, a estar fuera de casa, a estar sola, a las multitudes, a tener alguien detrás y temores sexuales.

Estas reacciones, complejas y variadas, conforman un patrón de respuesta normal y adaptado ante la agresión recibida. Sin embargo, Burgess y Holmstrom también distinguieron dos variantes de tipo patológico o desadaptativo. El primero, llamado de «reacción combinada», surgía en aquellas mujeres con una historia problemática a nivel físico y/o psicológico, o con unas deficiencias de relación interpersonal. En este caso las víctimas presentaban brotes psicóticos, intentos de suicidio, fuertes alteraciones psicosomáticas y depresión severa.

El segundo cuadro patológico, de «reacción silenciosa o inhibida», presentaba síntomas de ansiedad progresivamente mayores, largos períodos de silencio, malestar físico, una historia de reacciones fóbicas a estar sola o salir de casa, así como evitación de la actividad sexual. Estas mujeres frecuentemente habian sufrido con anterioridad una agresión sexual, en su niñez o adolescencia, y a menudo no habían hablado a nadie de ello, soportando esta carga a solas. Estas mujeres hablaban tanto de la violación anterior como de la actual.

El trabajo de Burgess y Holmstrom es ambicioso por cuanto abarca el estudio de la víctima en todas las facetas de su conducta. En este sentido, un aspecto de singular importancia radica en la reacción de padres, amigos, etc., ante el hecho. Brownmiller (1975, citado en Osborne, 1982) ha indicado que la relación marital puede venirse abajo al romperse el «sentido de la propiedad" existente en la misma, y contemplar el hecho delictivo de la violación como una infidelidad matrimonial (Weis y Weis, 1975).

Mas recientemente, Ellis, Atkeson y Calhoun (1981) han continuado investigando el trauma de la violación, pero, a diferencia de Burgess y Holmstrom, se preocuparon por analizar los efectos a largo plazo, es decir, después de haber transcurrido al menos un año desde la victimación. En el estudio de Ellis y colaboradores participaron 27 mujeres, la mayoría de clase media y de raza blanca. La media de tiempo desde que había transcurrido la violación era de tres años. Se empleó un grupo control de 26 mujeres, apareadas en variables de edad, raza, estatus socioeconómico y estado civil.

A los sujetos se les administró: 1) diferentes tests, entre los que se encontraban el Inventario de Depresión de Beck, la Escala de Miedos de Veronen y Kilpatrick (basada en la primitiva de Wolpe y Lang, con el añadido de 42 items relacionados con la violación y la violencia en general), la Escala de Ajuste Social de Weissman y Bothwell, y la lista de Acontecimientos Placenteros de MacPhillamy y Lewinshon; 2) una entrevista semiestructurada que evaluaba la historia y el funcionamientos actual del sujeto en varias áreas: trabajo, relaciones interpersonales, funcionamiento sexual y matrimonial, salud, uso de alcohol y droga, y ajuste y tratamiento psicológico, y 3) la entrevista sobre Información del Asalto, con la derivación de una puntuación en la escala de Brutalidad.

Los resultados no dejaron lugar a dudas: las mujeres victimizadas exhibieron una mayor incidencia de depresión, un menor disfrute de las actividades cotidianas, y un funcionamiento interpersonal más desajustado; igualmente, también mostraron más miedos y pesadillas que el grupo control. 
Con respecto a la Entrevista de Información sobre el Asalto, se hizo evidente que el conocimiento o no del agresor influía en la forma y gravedad de la agresión: la puntuación en brutalidad era mucho mayor en los casos de agresores desconocidos. Con objeto de observar las diferencias existentes entre los impactos derivados de un agresor conocido o desconocido, las respuestas de las víctimas divididas en estos dos grupos fueron comparadas en los tests administrados, surgiendo algunas diferencias dramáticas: las víctimas de agresores desconocidos tenían puntuaciones disfuncionales en todas las medidas.

Finalmente, en la entrevista aparecieron muchos problemas (en el grupo experimental) de relación con hombres (divorcios, rupturas...) y de índole sexual (frigidez, lesbianismo, promiscuidad). Evitación de la violencia y preservación de la integración corporal aparecieron como las preocupaciones focales de esas mujeres.

Aun cuando el tamaño de la muestra no permite hacer generalizaciones con garantías, no cabe duda de que los indicadores obtenidos por Ellis et al. (1981) merecen serios estudios de comprobación ulteriores.

\section{Elementos mediadores del impacto de la violación}

Pero no todos los trabajos se han centrado en evaluar la naturaleza y duración de los impactos de la violación sobre la víctima. Trabajos como los comentados anteriormente y algunos más (Kilpatrick, Veronen y Resick, 1979; Notman y Nadelson, 1976; Becker, Abel y Skinner, 1979; Feldman-Summers, Gordon y Meagher, 1979; Gager y Schurr, 1976; McCombie, 1976) no estudian el proceso por el cual llegan a ocurrir estas alteraciones. Precisamente para suplir esa laguna en la psicología de la violación, Norris y Feldman-Summers (1981) diseñaron una investigación con el propósito de averiguar si tales alteraciones se relacionan con algunos factores que se han revelado como importantes en este tipo de casos.

En efecto, los factores seleccionados por los autores han sido expuestos de manera precisa por la literatura psicológica. Es el caso de la existencia o no de una denuncia (Burgess y Holmstrom, 1974a y b; Feldman-Summers y Palmer, 1980), de la aparición de sentimientos de autoculpabilidad (Burgess y Holmstrom, 1974a), de la percepción por parte de la víctima de existencia de apoyo interpersonal (Tsai, Feldman-Summers y Edgar, 1979) y la severidad del asalto (trabajo ya comentado de Ellis et al., 1981). Los impactos psicológicos evaluados (variables dependientes) fueron sintomas psicomáticos, decremento cuantitativo y cualitativo de la conducta sexual y aislamiento.

En el estudio participaron 179 mujeres, víctimas de violación, con una media de edad de 27.5 años, siendo en su mayoría de raza blanca. El $83 \%$ de las violaciones habían ocurrido en los cinco años anteriores. Se construyó un cuestionario para evaluar las cuatro variables independientes y las tres dependientes.

Los análisis de regresión se aplicaron para todas las variables independientes, a excepción de la variable denuncia, que se trató separadamente. Los resultados de esta variable no evidenciaron efecto significativo alguno sobre los impactos psicológicos. En cuanto a las otras variables independientes: 
1) En relación a sintomas psicosomáticos: sólo severidad del asalto es un predictor significativo. Le siguió de cerca aunque sin alcanzar significación estadística los sentimientos de culpabilidad.

2) En relación a la frecuencia y satisfacción sexual, aunque $1 / 3$ de la muestra señaló un decremento en esta variable, ninguna fue significativa, lo que indicaba que los individuos utilizados aquí eran de poco valor en predecir los efectos psicosexuales.

3) En relación a los cambios en aislamiento, de los cuatro indicadores que conformaban esta variable, sólo dos fueron significativos: «ir sola a películas y conciertos", que se relacionaba con presencia de apoyo masculino, e «ir sola a los bares», lo cual se relacionaba con la presencia de apoyo femenino y los sentimientos de culpabilidad.

En la Tabla II aparece la relación de los indicadores que conformaban las variables dependientes, así como los porcentajes de antes y después del asalto sexual.

TABLA II

Porcentaje de victimas declarando síntomas psicosomáticos, cambios en reclusión y en frecuencia y satisfacción sexual

(Norris y Feldman-Summers, 1981)

\begin{tabular}{lcc}
\hline \multicolumn{1}{c}{ Impacto } & $\%$ antes & \% después \\
\hline Sintoma & & \\
Dificultades en dormir & 13,5 & 83,7 \\
Problemas de apetito & 19,2 & 63,8 \\
Cistitis & 7,4 & 18,0 \\
Irregularidades en el ciclo & 20,2 & 28,6 \\
Dolor de cabeza & 23,2 & 40,0 \\
Cambios de humor & 29,4 & 63,6 \\
Depresión & 30,4 & 79,6 \\
Excitabilidad & 24,1 & 42,9 \\
Libros frecuentes & 13,2 & 59,1 \\
Perder la paciencia & 18,6 & 42,7 \\
Reclusión (nunca salir sola) & & \\
Cines/conciertos & & 91,9 \\
Restaurantes & 65,7 & 75,4 \\
Bares & 41,1 & 89,5 \\
Lugares públicos & 71,3 & 64,7 \\
Conducta sexual & 28,6 & \\
& & \\
Frecuencia de sexo oral & No decrementa & Si decrementa \\
Frecuencia de coitos & & 25,5 \\
Frecuencia de orgasmos & 74,5 & 29,9 \\
Satisfacción sexual completa & 71,1 & 21,7 \\
\hline
\end{tabular}

Comentando los resultados, parece clara la relación aparecida entre severidad del asalto y problemas psicosomáticos. Por lo que respecta a la variable aislamiento, los datos muestran la importancia de contar con otros significativos prestando apoyo a la víctima. Las diferencias detectadas en relación a si este apoyo es proporcionado por hombres o mujeres pueden deberse al mayor riesgo implicado en cada una de las situaciones, donde la 
investigación se decanta a considerar que las mujeres víctimas de violación ponen más interés en la companía femenina. Y precisamente el «ir sola a los bares» denota mayor riesgo que el asistir a conciertos o películas; de ahí que la presencia de apoyo femenino pueda tener más peso en el caso primero (Norris y Feldman-Summers, 1981).

También en relación con la variable aislamiento resulta interesante constatar que las víctimas con sentimientos de culpabilidad no cambian dramáticamente su vida, ya que siguieron exponiéndose a situaciones de riesgo.

Finalmente, por lo que respecta a la conducta sexual, los resultados evidencian el fracaso a la hora de detectar con el instrumento empleado un declinar en la frecuencia y calidad de la experiencia sexual. Lo cierto es que contamos con algunas investigaciones, ya señaladas, indicando que efectivamente se produce tal deterioro sexual, si bien es posible que los actos relacionados con el asalto sexual produzcan tanto un decremento en las mujeres victimizadas como en aquellas que no han sufrido violación alguna (Orlando y Koss, 1983).

\section{La otra violación: la víctima en el proceso penal}

Con ser duros los momentos que acompañan a la agresión sexual y a su posterior ajuste, no acaban ahí las vicisitudes de la mujer violada. Paradójicamente, las instituciones legales someten a la víctima a todo un proceso arduo, y de efectos psicológicos graves. De ahí que Burgess y Holmstrom denominen todas esas circunstancias como la «segunda violación».

Antes de entrar en pormenores, es de sumo interés traer a colación aquí un artículo de Walshy Schram (1980), el cual aborda la problemática de las víctimas de la violación estableciendo un paralelismo con las víctimas de los delitos de cuello blanco, término introducido por Sutherland (1940) para referirse a aquellas acciones ilegales cometidas por gente de alto estatus, claramente delimitada de la población típicamente delictiva (delitos «duros» o comunes).

Este paralelismo se establece en tres puntos: en ambos casos la conducta proscrita no está suficientemente bien definida, se albergan dudas sobre si la víctima cooperó o no en el acto y, finalmente, en ambos casos se evidencia un componente devaluador con respecto a aquélla (del tipo «ella se lo buscó»). En concreto, Walsh y Schram señalan que el problema radica en la víctima, no en el agresor, y la percepción social que ella provoca.

En resumidas cuentas, nos encontramos con un problema de estereotipos, en donde el sistema legal no hace sino retomarlos en cada una de sus fases, con la consiguiente extendida impunidad para los agresores y consiguientemente traumatización de las víctimas.

En sendos artículos, Burgess y Holmstrom (Burgess y Holmstrom, 1974b, Holmstrom y Burgess, 1974), siguiendo con su estudio de seguimiento de las víctimas allegadas a la sala de urgencias del Hospital de Boston, documentaron con claridad los impactos psicológicos sobre las víctimas en las sucesivas fases del proceso legal. Veámoslos:

1) La denuncia. En los 61 casos estudiados por las autoras, sólo en el $25 \%$ de los mismos la decisión final procedió de la propia interesada. Mayormente alguien del círculo íntimo tomó la responsabilidad final. Este es un primer indicador del estado de dependencia en que se halla la mujer vio- 
lada. Socializada en la pasividad y protección del varón, ella es incapaz de tomar una decisión que va a exponer a la luz pública actos en donde su propia intimidad va a sufrir por segunda vez.

2) Interrogatorio policial de la víctima. En este punto el estereotipo supone una actitud antipática y escéptica por parte de la policía. Contrariamente, $2 / 3$ de la muestra señaló que èsta se había conducido de manera adecuada con ella. Pero, en cualquier caso, la investigación exhaustiva que se realiza (Chappell 1977b, citado en Bartol, 1980) requiere que la víctima recuerde y describa circunstancias escabrosas ante los funcionarios, fundamentalmente hombres.

3) Revisión médica. Posteriormente, ha de someterse a una cuidadosa revisión médica, para la comprobación de penetración y uso de la fuerza.

4) Decisión de presentar cargos. En este punto, 9 víctimas desistieron por temor a represalias de los allegados al agresor o a éste mismo, mientras que 8 no quisieron pasar por la exposición pública ante el juicio.

5) En el juicio, Burgess y Holmstrom (1974b) señalan 3 factores como fuentes principales de stress para la víctima: las demoras en su celebración, que obligan a la interesada a mantener la tensión previa; la exposición pública del hecho, donde ella ha de repetir cada detalle del incidente, así como otros hechos de su vida personal ante numerosos extraños ( A cada persona en el juicio se le da la oportunidad - al menos de segunda mano- para que sea testigo de la violación» pág. 27); y el hecho de que la víctima sea tratada como una delincuente.

Este último punto tiene especial interés. En el análisis de contenido que Burgess y Holmstrom (1974b) hicieron de los argumentos exhibidos en las 18 causas que finalmente se celebraron, los tópicos que solían emplearse eran los siguientes: ¿consintió la víctima? ¿Ofreció resistencia la víctima? ¿Cuál es su reputación y conducta sexual? ¿Estaba necesitada de dinero? $¿$ En que estado emocional se hallaba después del asalto? ¿Denunció la propia víctima la violación? ¿Ocurrió de verdad el acto sexual? ¿La denuncia fue una treta para escapar del castigo de alguien (padres, novio)? ¿Quería vengarse de alguien?

Es obvio que en estos casos se está empleando con la víctima un doble estándar de actuación. Por lo que respecta a si la mujer consintió en el encuentro sexual, la defensa ha de presentar la violación como un acto fundamentalmente sexual (placentero). Pero nosotros ya sabemos que se trata fundamentalmente de un acto agresivo. En este punto se recurre a estereotipos tales como que la mujer «estaba realmente pidiendo ser forzada".

En la mayoría de los otros argumentos se hace evidente cómo los «dobles estándares» entran en acción. Así, mientras el proceso de socialización exige a la mujer que 1) sea sumisa y no agresiva, 2) sea controlada en sus expresiones, y 3) parezca atractiva a los ojos de los hombres, en cambio durante el transcurso del juicio se le acusa como responsable del incidente si 1) no peleó hasta la extenuación, aun cuando ello hubiera puesto en peligro su vida, 2) si mantiene la serenidad como para recordar todos los hechos con minuciosidad, 3) si iba vestida de manera "socialmente atractiva" (Holmstrom y Burgess, 1974).

Los efectos psicológicos son «devastadores»: 
«El proceso recapitula desde el punto de vista psicológico la situación original de la violación. En la medida en que entra en la sala, los recuerdos del hecho se acentúan. Ella volverá a ver al acusado (...) Pero pronto su atención se dirige hacia el abogado defensor del acusado. Este llega a ser el asaltante en esta nueva arena. Previamente la mujer fue atacada físicamente (...) ahora va a ser atacada verbalmente. Es él, y no ella, quien conoce cómo funciona el juicio...” (Burgess y Holmstrom, 1974b).

A decir de las autoras, todo el entramado legal posibilita que la víctima viva en un estado de «tiempo suspendido", en donde «con frecuencia la mujer sólo intenta vivir de un día para otro» (pág. 29).

\section{COMENTARIO FINAL}

Desde nuestro punto de vista, parece claro que la psicología de la delincuencia tiene que ser más flexible e incluir entre sus metas una psicología de la víctima. En el caso de la violación resulta evidente cómo el proceso de victimación no acaba en la agresión sexual, sino que continúa en el medio social de la víctima y tiene una secuela negativa en el proceso penal por el que necesariamente ha de pasar.

Se trata, en definitiva, de mujeres "normales", con inquietudes sociales y personales, para las cuales la violación supone una grave disrupción a múltiples niveles. Ahora bien, si las víctimas no son mujeres «ligeras", los agresores sexuales no son hombres «desesperados» de sexo. El acto de la violación es un acto agresivo, el cual responde a unos roles y una ideología social que lo mantiene impune $y$, en este sentido, lo fomenta.

La existencia de tipologías con unos tipos definidos no nos aporta grran cosa a la comprensión de los fenómenos. Los estudios de Feldman-Summers y Malamuth son de gran interés por cuanto unen actitudes con posibles facilitadores fisiológicos, pero aun en este caso desconocemos el posible patrón causal. Así, puede ocurrir que la mayor responsividad sexual de los violadores se deba a una socialización o condicionamiento específico.

Hasta que no demostremos lo contrario, el papel actitudinal se erige como el más relevante en la génesis y fomento de este delito. Las medidas preventivas deberían dirigirse hacia una mayor informaión social de lo que supone la agresión sexual, desvelando los mitos y creencias erróneas. Esto debería incluir una educación sexual a todos los niveles, pero muy especialmente en los medios de comunicación social.

\section{Referencias}

Abel, G. G.; Barlow, D. H.; Blanchard, E. y Guild, D. (1977). The components of rapist' sexual arousal. Arch. of Gen., Psychiat., 34, 895-903.

AbEL, G. G.; BeCKER, J. V.; Blanchard, E. y DJENDEREDJIAN, A. (1978). Differentiating sexual aggresives with penile tumescence measures. Criminal Just. and Behavior, 5 , 315-322.

AbeL, G. G.; BlanChard, E. y BeCKer, J. V. (1976). Psychological treatment of rapist. En Walker y Brodsky (comp.): Sexual assault: The victim and the rapist. Lexington, Mass.: Lexington Books.

AmIR, M. (1971). Patterns in forcible rape. Chicago: Universidad de Chicago Press. 
ANNIS, L. V. (1982). A residential treatment program for male sex offenders. $1 . J$. Of Offender Ther., and Comp. Criminology, 26 (3), 223-235.

Barbaree, H. E.; MARShall, W. L y LANTHIER, R. D. (1979). Deviant sexual arousal in rapist. Beh. Resp. and Therapy, 17, 215-222.

BARLOw. (1974). The treatment of sexual deviation. En Calhoun, Adams y Mitchell (comp.): Innovative methods of treatment in psychopathology, Nueva Yor: Wiley.

BARTOL, C. (1980). Criminal behavior: a psychosocial approach. Prentice-Hall.

BAUERMEISTER, M. (1977). Rapist, victims and society. I. J. Offender Ther., and Comp. Criminology, 21 (3), 238-49.

BECKER, J. V; ABEL, G. G. y SKINNER, L. J. (1979). The impact of a sexual assault on the victim's sex life. Victimology, 4, 229-235.

BERNARD, T. J. (1981). The distinction between conflict and radical criminology. The J. of Crim. Law and Criminology, $72(1), 362-79$.

BRODSKY, S. L. (1980). Understanding and treating sexual offenders. The Howard J., XIX, $102-115$

BrohBERG, W. y COYLE, E. (1974). Rape: A compulsion to destroy. Med. Insight, 6 (4), 20-25.

BRownmiller, S. (1975). Against our will: men, women and rape. Nueva York: Simon and Schuster.

Burgess, A. W. y Holmstron, L. L. (1974a). Rape trauma syndrome. American Joumal of Psychiatry, 131, 981-986. [Trad. cast. 1979: Ed. Dédalos].

Burgess, A. W. y Holmstron, L. L. (1974b). Rape: the victim and the criminal justice system. En Drapkin y Viano (comp.): Victimology: a new focus. Health.

BURT, M. R. (1978). Attitudes supportive of rape in American culture. Washington: Gov. Printing Office.

BURT, M. R. (1980). Cultural myths and supports for rape. Journal of Personality and Social Psychology, 38, 217-230.

CHAPPELL, D. (1977a). Forcible rape: a national survey of the response by police. Washington: Gov. Printing Office.

CHAPPELL, D. (1977b). Forcible Rape: A National survey of the response by prosecurts. Washington, D.C.: U.S. Gov. Printing Office.

CheCK, J. V. P. y MALAmUTH, N. M. (1983). Sex role stereotyping and reactions to depictions of stranger versus acquaintance rape. Journal of Personality and Social Psychology, 45, 344-56.

Cohen, M. L.; Garofalo, R.; Boucher, R. y Seghorn, T. (1971). The psychology of rapists. Seminars in Psychiatry, 3, 307-27.

COHEN, M.; SEGHORN, T. y CALMAS, W. (1969). Sociometric study of the sex offender. Journal of Abnormal Psychology, 74, 249-255.

Drapkin, S. y VIANO, E. (1974). Victimology, Heath.

Ellis, E. M.; ATKESON, B. M. y CALHOUN, K. S. (1981). An assessment of longterm reaction to rape. Journal of Abnormal Psychology, 90 (3), 263-66.

Feldman-Summers, S.; Gordon, P. E. y MeaGHer, J. R. (1979). The impact of rape on sexual satisfaction. Joumal of Abnormal Psychology, 88 (1), 101-105.

FisheR, G. y RIVLIN, E. (1971). Psychological needs of rapist. B. J. Criminology, 11, 182-5.

FRENCH, L. A. y WAILES, S. N. (1982). Perceptions of sexual deviance. I. J. of Offender Therapy and C. Criminology, 26 (3), 242-49.

GAGER, N. y SCHURR, C. (1976). Sexual assault: Confronting rape in America. Nueva York: Grosset and Dunlap.

Goldstein, J. H. (1977). Aggression and crimes of violence. Nueva York: Oxford [Trad. cast. 1979 , ed. El manual moderno].

Groth, A. (1979). Men who rape. Nueva York, Plenum Press.

GROTH, A.; URGESS, A. W. y HOLMSTROM, L. L. (1977). Rape power, anger and sexuality. Am. J. of Psychiat., 134, 1239-1243.

Henn, F. A.; Herjanic, M. y Vanderpearl, R. H. (1976). Forensic Psychiatry: Profiles of two types of sex offenders. Am J. of Psychiat., 133, 694-696.

HolmSTROM, L. L. y BURGeSS, A. W. (1974). Rape. The victim goes on trial. En Drapkin y Viano (comp.): Victimology: a new focus, vol. 3 , Heath.

Karpman, B. (1954). The sexual offender and his offenses. Nueva York: Julia Press.

Kilpatric, D. G.; Veronen, L. J. y Resick, P. A. (1979). Assessment of the aftermath of rape: changing patterns of fear. Journal of Behavior Assessment, 1, 133-148.

Kopp, S. B. (1962). The character structure of sex offender. Am. J. of Psychoterapy, 16, 64-70.

MacDonald, J. (1974). Rape: offenders and theirs victims. Springfield, Thomas.

Malamuth, N. M. (1981a). Rape fantasies as a function of exposure to violent-sexual stimuli. Archives of sexual behavior, 10, 33-43.

Malamuth, N. M. (1981b). Rape proclivity among males. Journal of Social Issues, 37, 138-157.

Malamuth, N. M. y CheCK, J. V. P. (1980). Penile tumescence and perceptual responses to rape as a function of victim's perceived reactions. Joumal of Applied S. Psychology, 10, 528-547. 
Malamuth, N. M. y CheCK, J. V. P. (1980b). Sexual arousal to rape and consenting depictions: the importance of the woman's arousal. Journal of Abnormal Psychology, 89, 763-766.

Malamuth, N. M. y CHECK, J. V. P. (1983). Sexual arousal to rape depictions: Individual differences. Journal of Abnormal Psychology, 92, 55-67.

MaLAmUTH, N. M.; HABER, S. y FeShBACH, S. (1980). Testing hypothesis regarding rape: exposure to sexual violence, sex differences, and the "normality" of rapists. Journal of Res. in Personality, 14, 121-137.

McCALDON, R. J. (1967). Rape. Canadian Journal of Corrections, 9, 37-46.

McCORD, W. (1982). The psychopath and milieu therapy. Academic Press.

Nedoma, K.; Mellan, J. y Pondelickova, J. (1971). Sexual behavior and its development in pedophilic men. Archives of sexual behavior, 1, 267-71.

NORRIS, J. y FELDMAN-SUMMERS, S. (1981). Factors related to the psychological impacts of rape on the victim. Journal of Abnormal Psychology, 90 (6), 562-67.

NOtMAN, M. T. y NADELSON, C. C. (1976). The rape victim: Psychodinamic considerations. Am. Journal of Psychiatry, 133, 408-413.

Orlando, J. A. y KOOS, M. P. (1983). The effect of sexual victimization on sexual satisfaction: A study of the negative-Association hipothesis. Journal of Abnormal Psychology, 92 (1), 104-106.

OSBORNE, K. (1982). Sexual violence. En Feldman (comp.): Developments in the study of criminal behavior. Wiley.

PATH, A. R. (1976). The rapist in treatment proffesional myths and psychological realities. En Walker y Brodsky (comp.): Sexual assault: the victim and the rapist. Lexington, Mass., Lexington Books.

Peters, J. J. (1974). The Philadelphia rape victim study. En Drapkin y Viano (comp.): Victimology: a new focus. Heath.

QUINSEY, W. L.; ChAPLIN, T. C. y WARNEY, G. A. (1981). A comparison of rapists' and non sex offenders' sexual preferences for mutually consenting sex, rape, and physical abuse of women. Behavior Assessment, 3, 127-135.

RoEbuCK, J. B. (1967). Criminal typology. Springfield, I11.: Charles C. Thomas.

ROSENBERG. (1981). An empirical determination of sexual offenders subtypes. Convención anual de la A.P.A. Los Angeles.

SCUTT, J. A. (1982). To love, honour and rape myth impunity: wife as victim of rapen and the criminal law. En Schneider (comp.): The victim in international perspective. Walter de Gruynter.

Sutherland, E. (1949). White-Collar crimes. Nueva York: Dryden Press.

SUTHERLAN, S. y SCHERL, D. J. (1970). Patterns of response among victims of rape. Am. Journal of Orthopsychiat., 40, 503-511.

TSAI, M.; FELDMAN-SUMMERS, S. y EDGARD, M. (1979). Childhood molestation: variables related to differencial impacts on psychosexual functioning in adult women. Journal of $A b$ normal Psychology, 88, 407-417.

WALSH, M. E. y SCHRAM, D. D. (1980). The victim of White collar crime: accuser or accused? En Geis y Stodland (comp.): White Collar crime. Sage.

WolfGANG, M. (1967). Subculture of violence. En Wolfgang (comp.): Studies in Homicide. Nueva York: Harper and Row. 